\title{
The bright end of the exo-Zodi luminosity function: Disk evolution and implications for exo-Earth detectability
}

\author{
G. M. Kennedy and M. C. Wyatt \\ Institute of Astronomy, University of Cambridge, Madingley Road, Cambridge CB3 0HA, UK \\ email: gkennedy@ast.cam.ac.uk
}

\begin{abstract}
This contribution summarises the first characterisation of the $12 \mu \mathrm{m}$ warm dust ("exo-Zodi") luminosity function around Sun-like stars, focussing on the dustiest systems that can be identified by the WISE mission (Kennedy \& Wyatt 2013). We use the sample of mainsequence stars observed by Hipparcos within $150 \mathrm{pc}$ as an unbiased sample, and report the detection of six new warm dust candidates. The ages of five of these new sources are unknown, meaning that they may be sites of terrestrial planet formation or rare analogues of other old warm dust systems. We show that the dustiest old (> Gyr) systems such as BD+20 307 are 1 in 10,000 occurrences. Bright warm dust is much more common around young $(<120 \mathrm{Myr})$ systems, with a $\sim 1 \%$ occurrence rate. We show that a two component in situ model where all stars have initially massive warm disks and in which warm debris is also generated at some random time along the stars' main-sequence lifetime, perhaps due to a collision, can explain the observations. However, if all stars only have initially massive warm disks these would not be visible at Gyr ages, and random collisions on the main-sequence are too infrequent to explain the high disk occurrence rate for young stars. That is, neither component can explain the observations on their own. Despite these conclusions, we cannot rule out an alternative dynamical model in which comets are scattered in from outer regions because the distribution of systems with the appropriate dynamics is unknown. Our in situ model predicts that the fraction of stars with exo-Zodi bright enough to cause problems for future exo-Earth imaging attempts is at least roughly $10 \%$, and is higher for populations of stars younger than a few Gyr. This prediction of roughly $10 \%$ also applies to old stars because bright systems like BD+20 307 imply a population of fainter systems that were once bright, but are now decaying through fainter levels. Our prediction should be strongly tested by the Large Binocular Telescope Interferometer, which will provide valuable constraints and input for more detailed evolution models. A detection fraction lower than our prediction could indicate that the hot dust in systems like BD+20 307 has a cometary origin due to the quirks of the planetary dynamics. Population models of comet delivery need to be developed to help distinguish between different possible origins of warm dust.
\end{abstract}

Keywords. circumstellar matter, planetary systems

\section{Introduction}

Perhaps the most important long term goal of humankind is to find and communicate with aliens, the inhabitants of extra-Solar Systems. This goal is a difficult one, with many challenges to overcome before it is technically possible to even image an Earth-like planet around another star. When it is eventually attempted, imaging detection of these planets could still be thwarted by the presence of exo-Zodiacal dust located in or near the terrestrial zone, which with sufficient dust levels can mask the presence of exo-Earths (e.g. Beichman et al. 2006; Absil et al. 2010; Roberge et al. 2012). Therefore, in preparation for future missions that will target specific stars, a critical piece of information is whether those stars harbour dust at a level that precludes the detection of an Earth-like planet. 


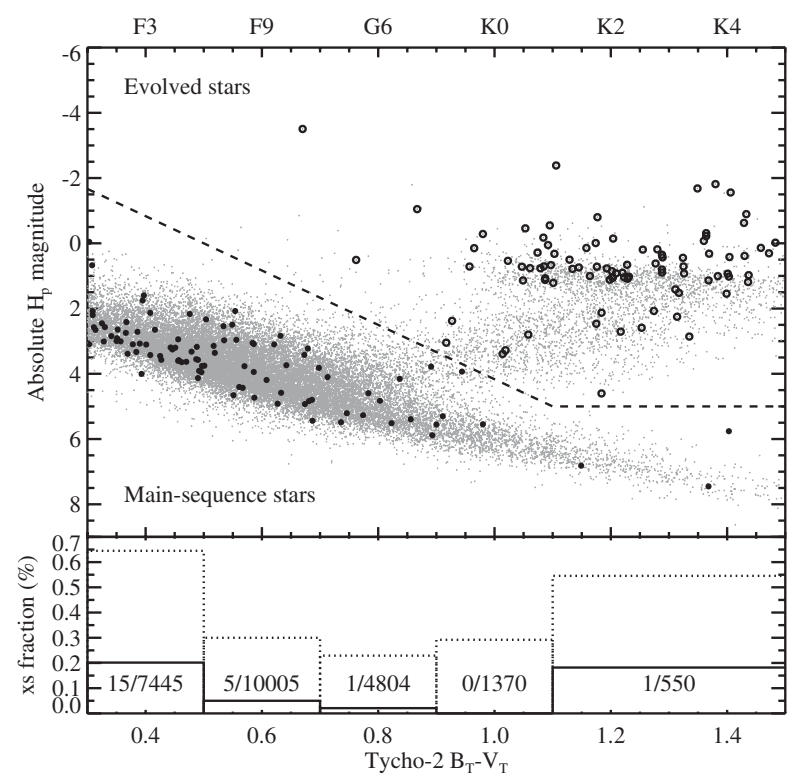

Figure 1. Top: HR diagram showing stars with $0.3<B_{T}-V_{T}<1.5$, split into Sun-like main-sequence stars and evolved stars by the dashed line. The top axis shows spectral types calculated using synthetic photometry of PHOENIX stellar atmosphere models (Brott \& Hauschildt 2005). Stars with $W 1-W 3$ greater than $0.1 \mathrm{mag}$ are shown as circles; open for evolved stars and filled for main-sequence stars. Bottom: Fraction of main-sequence stars with $W 1-W 3>0.1$. The dotted bars show all stars with $W 1-W 3>0.1$ (i.e. filled dots in the top panel), while the solid bars and numbers show those with plausible warm dust emission after individual checking.

This contribution briefly describes our study of the exo-Zodi luminosity function (Kennedy \& Wyatt 2013), which had several goals. First, the frequency of exo-Zodi detected as infra-red excesses at $12 \mu \mathrm{m}$ as a function of brightness (the so-called "luminosity function") was derived using data from the WISE mission. Second, these detections, which were necessarily at the bright end of the luminosity function, were used in conjunction with a simple evolution model to make predictions for the frequency of much fainter, but much more common exo-Zodi. In the interests of brevity, here we have omitted many details, and refer the reader to Kennedy \& Wyatt (2013) for a full description.

\section{Sample and warm dust candidates}

Stars are selected from the Hipparcos catalogue using the HR diagram shown in Figure 1. We use a fairly broad definition of Sun-like; $0.3<B_{T}-V_{T}<1.5$. We exclude evolved stars, which may show excesses due to circumstellar material associated with mass loss. Because they are selected solely using optical photometry and parallax, the 24,174 stars selected by these criteria are unbiased with respect to the presence of warm dust. Figure 1 shows all 27,333 stars within the $B_{T}-V_{T}$ range, the cut used to exclude evolved stars (dashed line), and those found to have $12 \mu \mathrm{m}$ excesses (large dots and circles).

Not all stars with apparent $12 \mu \mathrm{m}$ emission represent a true indication of warm dust, so all 96 excess systems were vetted by inspecting the WISE images and constructing spectral energy distributions to check for consistency among the photometry (notes on individual systems are given in the appendix of Kennedy \& Wyatt 2013). Most were found to be spurious due to image contamination and/or poor flux measurement, leaving 22 warm dust candidate systems, all but seven of which were previously known. 


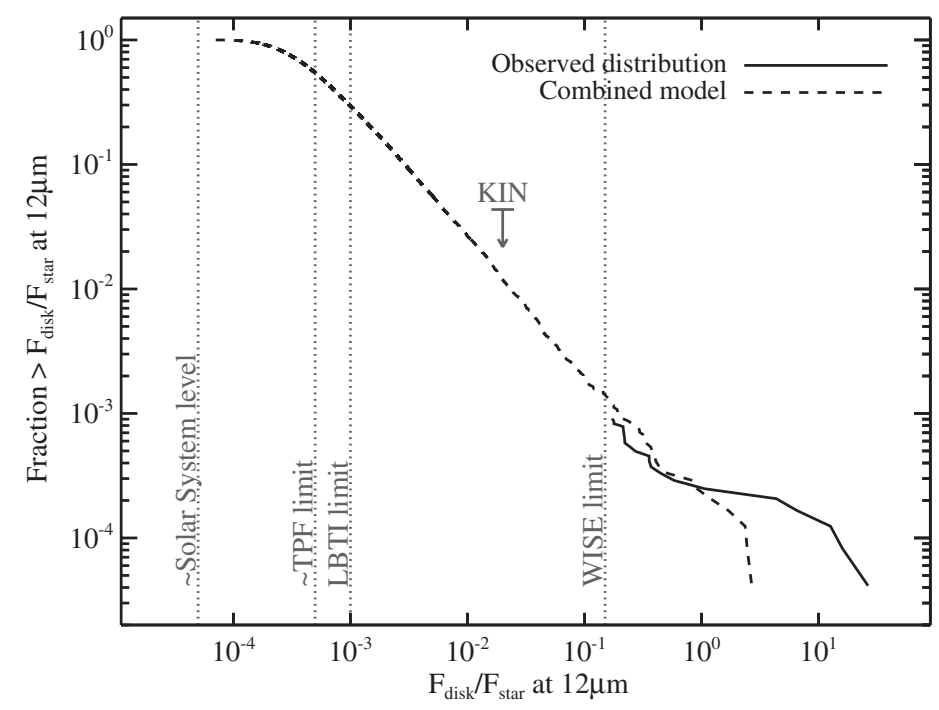

Figure 2. Observed luminosity function (solid line) and a simple prediction for faint exo-Zodi for stars between 0 and $13 \mathrm{Gyr}$ with a single realisation of a Monte-Carlo population model of evolution. Grey dotted lines show the limits for LBTI and WISE sensitivity, as well as approximate levels for the Solar System Zodiacal cloud and for which TPF-like missions will be compromised by exo-zodiacal dust. The upper limit from the 23-star KIN survey is also shown.

\section{Excess selection and warm dust candidates}

If warm dust is sufficiently bright relative to the host star, it will manifest as a detectable mid-infrared (i.e. $10-20 \mu \mathrm{m}$ ) excess relative to the stellar photosphere, which for $\sim 300 \mathrm{~K}$ dust peaks near $12 \mu \mathrm{m}$. The WISE bands are well suited for this task, and because it has about an order of magnitude better sensitivity to $300 \mathrm{~K}$ dust compared to W4 (see Fig 2 of Kennedy \& Wyatt 2012) we use the $12 \mu \mathrm{m} \mathrm{W3}$ band. $\dagger$

Our luminosity function is therefore formally the distribution of $12 \mu \mathrm{m}$ disk-to-star flux density ratios, which we will use in cumulative form. We use the term "luminosity function" largely for conciseness. The disk luminosity of course not only depends on the $12 \mu \mathrm{m}$ flux ratio, but also the disk temperature and stellar properties.

Figure 2 shows the $12 \mu \mathrm{m}$ luminosity function derived using the WISE data and one realisation of a Monte-Carlo population model of evolution. The model under-predicts the number of brightest excesses, but varies considerably due to the small number of such disks. Below $F_{\text {disk }} / F_{\star} \sim 0.1$ all realisations, and hence the predictions for fainter disks, are very similar.

The right side of the figure is covered by photometric surveys like the one presented here, whereas fainter disks (left side) can only be detected with more sophisticated methods, such as those employed by the KIN and LBTI. We have taken the approximate Solar System $12 \mu \mathrm{m}$ dust level as $F_{\text {disk }} / F_{\star}=5 \times 10^{-5}$, and the LBTI sensitivity as 20 times this level (Hinz 2009). The predicted limit for exo-Earth detection by a Terrestrial Planet Finder (TPF)-like mission is also shown (at ten times the Solar System level).

The predicted disk fraction is about $50 \%$ at the LBTI sensitivity. An unbiased LBTI survey of just a few tens of stars would therefore result in a strong test of our prediction, and has the potential for detection of a dozen or so disks if the evolution predicted by

$\dagger$ The WISE bands are known as $W 1, W 2, W 3$, and $W 4$, with wavelengths of $3.4,4.6,12$, and $22 \mu \mathrm{m}$ respectively (Wright et al. 2010; Jarrett et al. 2011). 
our model is correct. Such a result would be a great success for the instrument, but less promising for the study of exo-Earths; another prediction of our model is that roughly only a few tens of percent of stars are amenable to exo-Earth detection if our combined model is correct.

\section{Summary and Conclusions}

We have presented an analysis of the bright end of the $12 \mu \mathrm{m}$ warm dust luminosity function as seen by WISE around nearby Sun-like stars. We report the possible detection of 6 new warm dust candidates, HD 19257, HD 23586, HD 94893, HD 154593, HD 165439, and HD 194931.

There is a clear preference for excess emission around young stars, with at least 14/22 systems younger than $120 \mathrm{Myr}$, despite the $\sim 10 \mathrm{Gyr}$ stellar lifetime. However, two of the dustiest systems are $\sim 1-2$ Gyr old, and another may be $\sim$ Gyr old, suggesting that while youth is an important factor for setting the brightness of warm dust, it is not the sole factor.

Using a simple in situ evolution model to interpret the observed luminosity function, we show that neither a picture where all systems have initially massive disks, nor one where all excesses are due to randomly timed collisions, can explain the fact that warm dust is observed around both young and old stars. A simple combination of these two in situ scenarios reproduces the observed luminosity function. However, we cannot rule out that warm dust is in fact dominated by comet delivery. In this scenario comets are scattered from a more distant reservoir, and reliable luminosity function predictions cannot be made because they depend on the distribution of systems with the appropriate dynamics.

We have made simple predictions for the number of exo-Zodi that might be detected by an unbiased LBTI survey. For the expected sensitivity we anticipate a handful of detections and a strong test of our evolution model. Such tests are a crucial step towards understanding the origins and evolution of exo-Zodi, and ultimately the imaging of Earthlike planets.

\section{References}

Absil, O. et al. 2010, in Astronomical Society of the Pacific Conference Series, Vol. 430, Pathways Towards Habitable Planets, ed. V. Coudé Du Foresto, D. M. Gelino, \& I. Ribas, 293

Beichman, C. A. et al. 2006, ApJ, 652, 1674

Brott, I. \& Hauschildt, P. H. 2005, in ESA Special Publication, Vol. 576, The Three-Dimensional Universe with Gaia, ed. C. Turon, K. S. O'Flaherty, \& M. A. C. Perryman, 565

Hinz, P. M. 2009, in American Institute of Physics Conference Series, Vol. 1158, American Institute of Physics Conference Series, ed. T. Usuda, M. Tamura, \& M. Ishii, 313-317

Jarrett, T. H. et al. 2011, ApJ, 735, 112

Kennedy, G. M. \& Wyatt, M. C. 2012, MNRAS, 426, 91

-. 2013, MNRAS

Roberge, A. et al. 2012, PASP, 124, 799

Wright, E. L. et al. 2010, AJ, 140, 1868

\section{Discussion}

S. Metchev: In our search for W3 and W4 excesses in WISE (see Patel \& Metchev poster) we found that $75 \%$ of our W3 excesses have even stronger W4 excesses. That is, they do not seem to be exo-Zodi. Do you have estimates of temperatures for your detections? 
KENNEDY: Yes, most of our stars have W4 detections so we can determine temperatures. These are given in Kennedy \& Wyatt (2013) and are typically a few hundred Kelvin. We also have some discussion of temperatures in Section 4.2 .

C. STARK: You began your talk by listing the different mechanisms that could produce exo-Zodiacal dust. It's not unreasonable to think that different mechanisms may dominate at different ages and/or dust levels. So is it valid to extrapolate measurements of dense disks associated with young stars to lower mass disks around older stars?

KEnNEDY: This is a very good point. The model we used for the prediction is based on the assumption that disks decay collisionally, and there is no specification of what the mechanism is that starts the evolution. So in this sense the model is OK because the mechanism doesn't matter as long as the subsequent evolution is something like our model. However, as we discuss in our paper, comet delivery is an equally viable cause of exo-Zodi, and it could be that this works preferentially at young ages and is less important at late times. If this were the main cause of exo-Zodi then the extrapolation could be very different. Making any kind of prediction for this scenario is extremely dependent on the assumptions made, so we chose to only model the collisional scenario as it is easier to define.

S. ERTEL: In the context of the model random collisions are needed. Is it possible to constrain how frequent these collisions have to be in order to reproduce the detection rate you presented (e.g. once in each system per lifetime, more often)?

KENNEDY: In a crude way yes we can. There is a degeneracy between the number of collisions during a stars' lifetime and the decay rate after that collision. But if the decay rate is the same as needed for the disks that start decaying early on, the number of collions per stellar lifetime is just one. It's not a great constraint but is probably indicative if this scenario is correct. 